\title{
BMJ Open Cohort profile: the Netherlands Cohort Study on Acute HIV infection (NOVA), a prospective cohort study of people with acute or early HIV infection who immediately initiate HIV treatment
}

\author{
Maartje Dijkstra (1) , ${ }^{1,2}$ Henrieke Prins (D) , ${ }^{3}$ Jan M Prins, ${ }^{2}$ Peter Reiss, ${ }^{2,4,5}$ \\ Charles Boucher, ${ }^{6}$ Annelies Verbon, ${ }^{3}$ Casper Rokx, ${ }^{3}$ Godelieve de Bree ${ }^{7}$
}

To cite: Dijkstra M, Prins H, Prins JM, et al. Cohort profile: the Netherlands Cohort Study on Acute HIV infection (NOVA), a prospective cohort study of people with acute or early HIV infection who immediately initiate HIV treatment. BMJ Open 2021;11:e048582. doi:10.1136/ bmjopen-2020-048582

- Prepublication history for this paper is available online. To view these files, please visit the journal online (http://dx.doi. org/10.1136/bmjopen-2020048582).

CB deceased.

$\mathrm{MD}$ and $\mathrm{HP}$ are joint first authors.

$\mathrm{CR}$ and $\mathrm{GdB}$ are joint senior authors.

Received 03 January 2021 Accepted 14 October 2021

D) Check for updates

(c) Author(s) (or their employer(s)) 2021. Re-use permitted under CC BY-NC. No commercial re-use. See rights and permissions. Published by BMJ.

For numbered affiliations see end of article.

Correspondence to Maartje Dijkstra; maartje.dijkstra@ amsterdamumc.nl

\section{ABSTRACT}

Purpose Initiation of combination antiretroviral therapy (CART) during acute or early HIV-infection (AEHI) limits the size of the viral reservoir and preserves immune function. This renders individuals who started CART during AEHI promising participants in HIV-cure trials. Therefore, we established a multicentre prospective cohort study in the Netherlands that enrols people with AEHI. In anticipation of future cure trials, we will longitudinally investigate the properties of the viral reservoir size and HIV-specific immune responses among cohort participants.

Participants Participants immediately initiate intensified CART: dolutegravir, emtricitabine/tenofovir and darunavir/ ritonavir (DRV/r). After 4 weeks, once baseline resistance data are available, DRV/r is discontinued. Three study groups are assembled based on the preparedness of individuals to participate in the extensiveness of sampling. Participants accepting immediate treatment and follow-up but declining additional sampling are included in study group 1 ('standard') and routine diagnostic procedures are performed. Participants willing to undergo blood, leukapheresis and semen sampling are included in study group 2 ('less invasive'). In study group 3 ('extended'), additional tissue (gut-associated lymphoid tissue, peripheral lymph node) and cerebrospinal fluid sampling are performed.

Findings to date Between 2015 and 2020, 140 individuals with $\mathrm{AEH}$ have been enrolled at nine study sites. At enrolment, median age was 36 (IQR 28-47) years, and $134(95.7 \%)$ participants were men. Distribution of Fiebig stages was as follows: Fiebig I, 3 (2.1\%); II, 20 (14.3\%); III, 7 (5.0\%); IV, 49 (35.0\%); V, 39 (27.9\%); VI, 22 (15.7\%). Median plasma HIV RNA was 5.9 (IQR 4.7-6.7) log10 copies/mL and CD4 count 510 (IQR 370-700) cells/ $\mathrm{mm}^{3}$. Median time from cART initiation to viral suppression was 8.0 (IQR 4.0-16.0) weeks.

Future plans The Netherlands Cohort Study on Acute HIV infection remains open for participant enrolment and for additional sites to join the network. This cohort provides a unique nationwide platform for conducting future in-depth virological, immunological, host genetic and interventional studies investigating HIV-cure strategies.

\section{Strengths and limitations of this study}

- Initiation of combination antiretroviral therapy (CART) during acute or early HIV infection (AEHI) limits the size of the viral reservoir and preserves immune function.

- The Netherlands Cohort Study on Acute HIV infection (NOVA) is a national prospective cohort study among participants with AEHI who immediately start CART.

- NOVA provides a platform for gaining detailed insight into participants' viral reservoir size and composition and their HIV-specific immune responses before and during treatment, and for conducting future interventional studies aimed at achieving post-treatment control and cure.

- Together now almost half of all cohort participants have consented to extended blood and tissue sampling, allowing us to longitudinally characterise the viral reservoir and the properties of the HIV-specific immune response in great detail.

- We will continue to focus on accelerated diagnostic and referral pathways in the earliest AEHI Fiebig stages I and II, which is particularly challenging due to the short duration of these stages.

\section{INTRODUCTION}

Despite the availability of combination antiretroviral therapy (cART), worldwide approximately 1.7 million people newly acquired HIV in 2019, 690000 people died of AIDS and onethird of people living with HIV (PLWH) still did not have access to cART. ${ }^{1}$ Further expansion of access to cART alone will not be sufficient to address these gaps, as universal cART has only led to a modest decrease in HIV incidence in certain high incidence regions and stigma remains a major barrier to HIV care, leading to reduced access and adherence to cART. $^{2-4}$ Furthermore, PLWH on cART may continue to experience a substantial physical and psychosocial burden. ${ }^{56}$ These data 


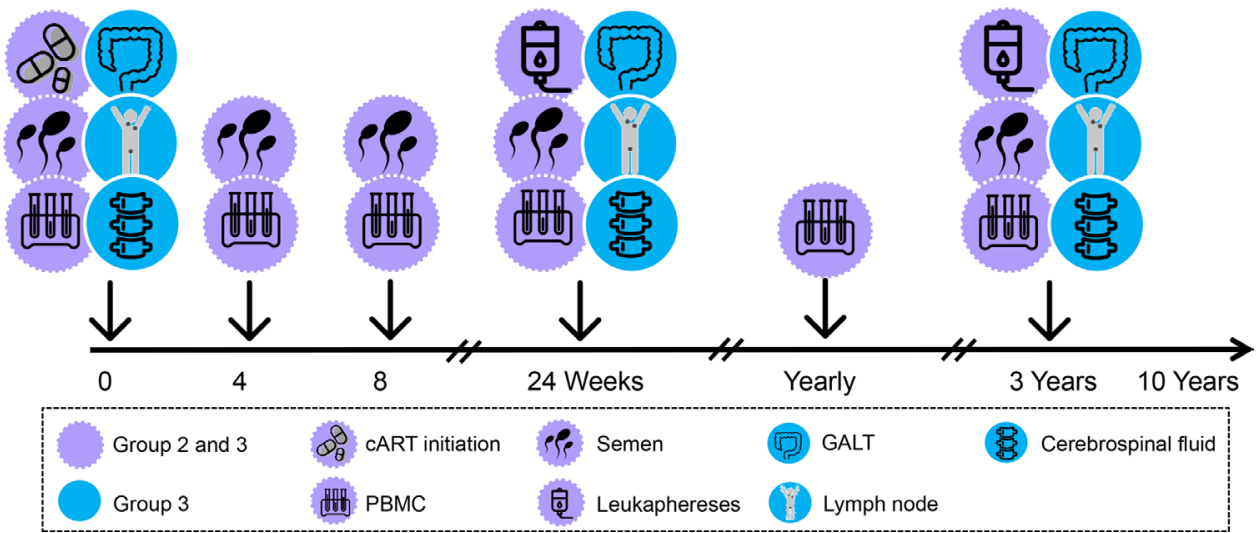

Figure 1 Overview of NOVA cohort study procedures for study groups 2 and 3. cART, combination antiretroviral therapy; GALT, gut-associated lymphoid tissue; PBMC, peripheral blood mononuclear cells.

illustrate the need to develop therapeutic strategies that lead to HIV cure. ${ }^{7}$ HIV cure is defined as a therapeutic strategy resulting in prolonged viral suppression in the absence of cART. There are several essential elements involved in the design of potentially favourable cure strategies. First, insight in viral and host-immune factors that are associated with viral control in the absence of cART is needed. Important in this respect is the observation that people who start cART in the early phase of infection potentially have the greatest possibility to benefit from such an intervention. ${ }^{8}$ Second, there is increasing insight, which indicates that the properties of the viral reservoir and the concomitant HIV-specific immune response are important factors determining the clinical outcome of cure interventions. The establishment of cohorts that have been well characterised with respect to viral and immunological properties is therefore pivotal to study potential cure strategies. ${ }^{9}$

cART suppresses plasma viraemia to below detection limits in the vast majority of PLWH, ${ }^{10}$ but is not able to clear the virus completely and an intracellular latent viral reservoir persists. Within the first hours following HIV infection, a viral reservoir is established, ${ }^{11}$ which is mainly localised in peripheral $\mathrm{CD} 4^{\text {pos }} \mathrm{T}$ cells and lymphoid tissues. ${ }^{12-16}$ The presence of the viral reservoir is responsible for a rapid rebound of viraemia when cART is interrupted. ${ }^{1718}$ However, when cART is initiated during acute or early HIV infection (AEHI; here defined as the first 6 months after infection), a select group of individuals is able to remain aviraemic after treatment interruption. This phenomenon is often referred to as post-treatment control or HIV remission. Studies suggest that this rare event of viral control related to treatment initiation during AEHI is associated with a significantly smaller viral reservoir. ${ }^{819-25}$ The relatively minor proportion of individuals that achieve post-treatment control together with the results from a seminal study that showed viral rebound in individuals that initiated cART in the earliest stage of infection, ${ }^{26}$ indicate that early containment of the viral reservoir by cART is only partially related to viral control.

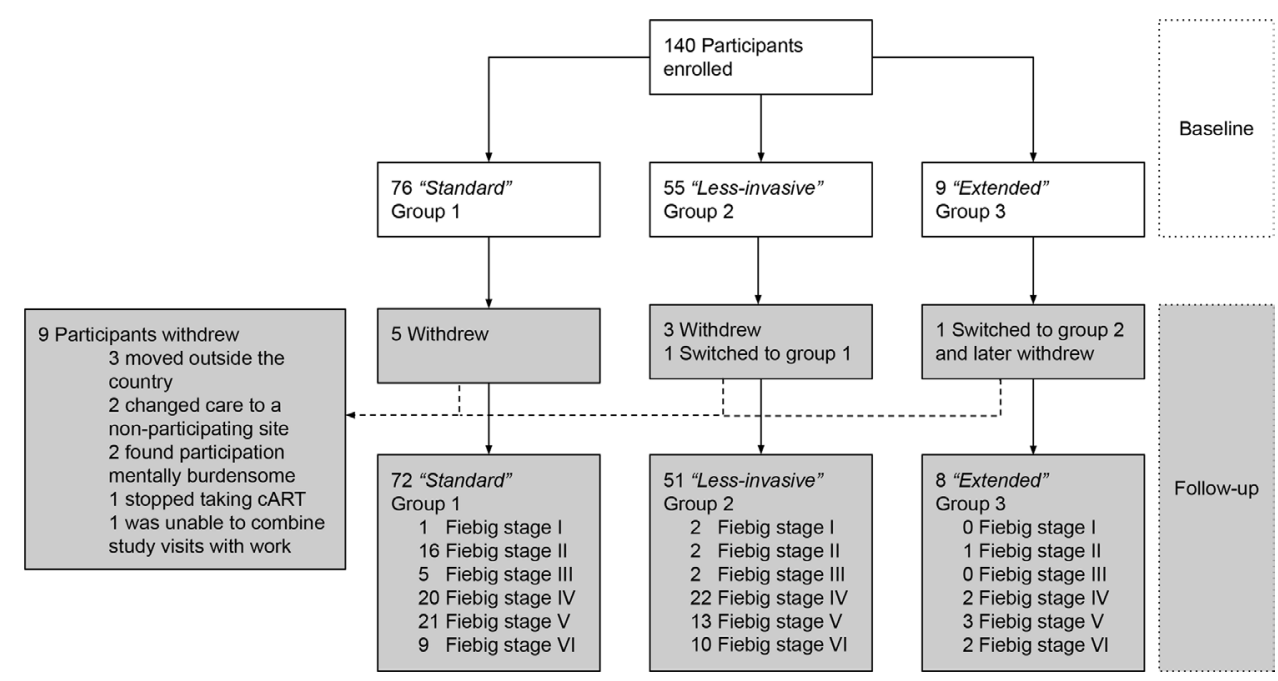

Figure 2 Disposition of The Netherlands Cohort Study on Acute HIV infection cohort study participants, August 2015 to July 2020. 'Standard' group 1 includes immediate combination antiretroviral therapy initiation and routine monitoring; 'Less invasive' group 2 includes routines monitoring, blood sampling for peripheral blood mononuclear cells and virological analyses, leukapheresis and semen sampling. 'Extended' group 3 includes group 2 sampling procedures and gut-associated lymphoid tissue, peripheral lymph node and cerebrospinal fluid sampling. 
Table 1 Baseline characteristics of first 140 The Netherlands Cohort Study on Acute HIV infection cohort study participants, August 2015 to July 2020

\begin{tabular}{|c|c|c|}
\hline & $\mathrm{n}$ or median & $\%$ or IQR \\
\hline Age in years & 36 & $28-47$ \\
\hline \multicolumn{3}{|l|}{ Gender } \\
\hline Male & 134 & $95.7 \%$ \\
\hline Female & 4 & $2.9 \%$ \\
\hline Transgender female & 2 & $1.4 \%$ \\
\hline \multicolumn{3}{|l|}{$\mathrm{MSM}^{*}$} \\
\hline Yes & 124 & $92.5 \%$ \\
\hline No & 10 & $7.5 \%$ \\
\hline \multicolumn{3}{|l|}{ Site of HIV diagnosis } \\
\hline STI clinic & 76 & $54.3 \%$ \\
\hline General practice & 39 & $27.9 \%$ \\
\hline Hospital & 19 & $13.6 \%$ \\
\hline Othert & 6 & $4.3 \%$ \\
\hline \multicolumn{3}{|l|}{ Year of enrolment } \\
\hline 2015 & 11 & $7.9 \%$ \\
\hline 2016 & 32 & $22.9 \%$ \\
\hline 2017 & 37 & $26.4 \%$ \\
\hline 2018 & 35 & $25.0 \%$ \\
\hline 2019 & 21 & $15.0 \%$ \\
\hline 2020 & 4 & $2.9 \%$ \\
\hline \multicolumn{3}{|l|}{ Fiebig stage $\neq$} \\
\hline 1 & 3 & $2.1 \%$ \\
\hline II & 20 & $14.3 \%$ \\
\hline III & 7 & $5.0 \%$ \\
\hline IV & 49 & $35.0 \%$ \\
\hline V & 39 & $27.9 \%$ \\
\hline VI & 22 & $15.7 \%$ \\
\hline
\end{tabular}

Resistance mutations*

$\begin{array}{lrl}\text { Yes } & 19 & 13.7 \% \\ \text { No } & 120 & 86.3 \% \\ \text { Type of resistance mutations§ } & & \\ \text { NNRTI resistance } & 7 & - \\ \text { NRTI resistance } & 15 & - \\ \text { PI resistance } & 1 & -\end{array}$

*1 missing value.

†Including community-based testing $(n=4)$, own initiative $(n=2)$ $\ddagger$ At cART initiation.

$\S 3$ participants had two mutations, therefore, the percentages are not reported.

ПM46L, not associated with darunavir/ritonavir resistance.

MSM, men who have sex with men; NNRTI, non-nucleoside reverse transcriptase inhibitor; NRTI, nucleoside reverse transcriptase inhibitor; PI, protease inhibitor; STI, sexually transmitted infection.

The HIV-specific T-cell response importantly contributes to the control of viral replication. ${ }^{86-28}$ The effect of early start of cART on the immune response can be discerned from the few studies in which participants were treated during AEHI and subsequently interrupted treatment. In the Viro-Immunological Sustained CONtrol after Treatment Interruption (VISCONTI) study, participants who were temporarily treated during early infection and then discontinued cART, post-treatment control occurred in 14 participants and was associated with a lower level of T-cell activation after discontinuation of cART. ${ }^{8}$ A recent study in which participants initiated cART during even earlier stages of infection (Fiebig stages I, II and III) showed that, before the start of cART, very early $\mathrm{CD} 8^{\text {pos }}$ $\mathrm{T}$ cells (in Fiebig stages I and II) had a memory signature, whereas later $\mathrm{CD} 8^{\text {pos }} \mathrm{T}$ cells (in Fiebig stage III) were equipped with effector function. ${ }^{27}$ Furthermore, in participants that started cART very early (Fiebig I), CD8 $8^{\text {pos }}$ $\mathrm{T}$ cells before the start of cART had less breadth and a low activation state ${ }^{26}$ and treatment resulted in enhanced effector function and less exhaustion. ${ }^{28}$

Taken together, these studies show that early cART reduces inflammation and improves T-cell function, but at least some viral exposure may be needed to drive the development of a potent $\mathrm{CD} 8^{\text {pos }} \mathrm{T}$-cell response.

As mentioned, individuals who start cART early in the course of infection have a potentially good starting point to benefit from cure interventions given their relatively small reservoir size and potentially a potent HIV-specific immune response, both of which depend on the Fiebig stage during which they initiated cART. The Netherlands Cohort Study on Acute HIV infection (NOVA) was initiated in 2015. This is a national prospective cohort study among participants with AEHI who immediately start cART. NOVA provides a platform for gaining detailed insight into participants' viral reservoir size and composition and their HIV-specific immune responses before and during treatment, in anticipation of future clinical trials aimed at HIV cure.

\section{COHORT DESCRIPTION}

\section{Study setting}

NOVA was initiated in August 2015 as an ongoing multicentre prospective cohort study enrolling participants diagnosed with AEHI who immediately initiate cART on diagnosis. Participants currently are enrolled in nine HIV treatment centres across the Netherlands (Amsterdam University Medical Centers (Academic Medical Center site), Amsterdam; Erasmus University Medical Center, Rotterdam; Maasstad Hospital, Rotterdam; Onze Lieve Vrouwe Gasthuis, Amsterdam; DC Klinieken, Amsterdam; University Medical Center, Utrecht; Radboud University Medical Center, Nijmegen; Leiden University Medical Center, Leiden and Rijnstate Hospital, Arnhem).

\section{Study population}

The NOVA cohort study enrols individuals who are (1) 18 years or older; (2) diagnosed with AEHI (defined according to Fiebig staging at the time of diagnosis ${ }^{29}$ and (3) willing to initiate cART within 24 hours of enrolment. Acute infection (Fiebig stages I-II) is defined as either plasma HIV RNA detectable by reverse transcription (RT)-PCR or HIV p24 antigen detectable by fourth generation ELISA without detectable anti-HIV antibodies. Early 
Table 2 Clinical and laboratory parameters of The Netherlands Cohort Study on Acute HIV infection cohort study participants stratified by Fiebig stage, August 2015 to July 2020

\begin{tabular}{|c|c|c|c|c|c|c|c|c|}
\hline & \multicolumn{2}{|c|}{ All participants $(n=140)$} & \multicolumn{2}{|c|}{ FI-II (n=23) } & \multicolumn{2}{|c|}{ FIII-IV ( $n=56)$} & \multicolumn{2}{|c|}{ FV-VI $(n=61)$} \\
\hline & Median & IQR & Median & IQR & Median & IQR & Median & IQR \\
\hline $\begin{array}{l}\text { Baseline plasma HIV RNA } \\
\text { (log10 copies } / \mathrm{mL} \text { ) }\end{array}$ & $5.9^{*}$ & $4.7-6.7$ & $6.4 \dagger$ & $5.0-7.0$ & 6.4 & $5.5-7.0$ & $5.0 \dagger$ & $4.5-5.9$ \\
\hline $\begin{array}{l}\text { Baseline CD4 } 4^{\text {pos }} \text { T-cell } \\
\text { count }\left(\text { cells } / \mathrm{mm}^{3}\right)\end{array}$ & $510 †$ & $360-700$ & $500 \dagger$ & $330-580$ & 480 & $325-620$ & 560 & $422-730$ \\
\hline $\begin{array}{l}\text { Baseline CD8 }{ }^{\text {pos }} \text { T-cell } \\
\text { count }\left(\text { cells } / \mathrm{mm}^{3} \text { ) }\right.\end{array}$ & $940 \dagger$ & $540-1430$ & $540 \dagger$ & $300-790$ & 795 & $465-1300$ & 1100 & $809-1590$ \\
\hline $\begin{array}{l}\text { Baseline CD4/CD8 T-cell } \\
\text { ratio }\end{array}$ & $0.55 \dagger$ & $0.37-0.96$ & $1.02 \dagger$ & $0.53-1.76$ & 0.6 & $0.36-1.01$ & 0.5 & $0.29-0.73$ \\
\hline $\begin{array}{l}\text { Days from HIV diagnosis } \\
\text { to cART initiation }\end{array}$ & 1 & $0-7$ & 1 & $0-1$ & 1 & $0-5$ & 3 & $0-9$ \\
\hline $\begin{array}{l}\text { Weeks from cART } \\
\text { initiation to viral } \\
\text { suppression } \ddagger \S\end{array}$ & $8.0^{\star}$ & $4.0-16.0$ & $10.0^{\dagger}$ & $5.3-20.0$ & 8.3 & $4.1-16.1$ & $7.7 \dagger$ & $4.0-12.0$ \\
\hline
\end{tabular}

\section{*2 missing values.}

†Missing value.

$\ddagger$ Assessed with Kaplan-Meier estimates.

§Defined as the first documented plasma HIV RNA $<40$ copies $/ \mathrm{mL}$.

IAmong 106 participants with a CD4/CD8 T-cell ratio of $<1$ at enrolment (10 participants with FI-II, 41 participants with FIII-IV, 55 participants with $\mathrm{FV}-\mathrm{VI})$.

${ }^{\star *}$ After 3 years of follow-up, less than $75 \%$ of participants achieved a CD4/CD8 T-cell ratio $\geq 1$, therefore it is not possible to estimate the $75^{\text {th }}$ percentile.

cART, combination antiretroviral therapy; F, Fiebig stage.

infection (Fiebig stages III-VI) is defined as plasma HIV RNA detectable by RT-PCR and with anti-HIV antibodies detectable by fourth generation ELISA in the presence of a negative, indeterminate or positive western blot. Individuals with a positive western blot are only included if their western blot is p31 negative or if they have a documented negative HIV test, 6 months prior to their HIV diagnosis.

\section{Study design}

Three study groups are assembled based on the preparedness of individuals to participate in the extensiveness of sampling (figure 1). Participants that accept immediate treatment and follow-up but decline additional blood and tissue sampling are included in study group 1 ('standard') and only routine diagnostic and follow-up procedures are performed. Participants willing to undergo blood sampling for peripheral blood mononuclear cells (PBMC) and virologic analyses, leukapheresis and semen sampling are included in study group 2 ('less invasive'). In study group 3 ('extended'), additional tissue (gutassociated lymphoid tissue, peripheral lymph node) and cerebrospinal fluid sampling are performed. In participants who provide written informed consent to participate in NOVA, samples are obtained at the time of enrolment and at several subsequent time points to analyse the size and characteristics of the viral reservoir and the immune response. Study participants are followed for at least 10 years and are allowed to change between groups if they wish so at any time point.

\section{Participant selection and recruitment}

Individuals diagnosed with AEHI who are referred for cART initiation to one of the participating study sites are informed about the study by their treating physician. The screening takes place within 24 hours after the study team has been made aware of a potential AEHI case. AEHI diagnosis can take place at a sexually transmitted infection (STI) clinic, general practice or hospital. STI clinics in the Netherlands routinely offer fourth generation ELISA antigen/antibody testing free of charge to men who have sex with men (MSM) and other high-incidence populations. These groups are advised to test for HIV every 3 months, in case of AEHI/STI symptoms or after they have been notified for HIV by a sexual partner. HIV-RNA testing by RT-PCR is only conducted if AEHI is highly suspected. For example, at the Amsterdam STI clinic this is offered to MSM with a high score on the 'Amsterdam score', an AEHI screening score, which includes AEHI symptoms and behavioural factors. ${ }^{30}$ General practitioners and hospitals usually provide fourth generation ELISA antigen/antibody testing to people with AEHI/STI symptoms. In Rotterdam, the STI clinics, general practitioners and hospitals provide point-of-care HIV-RNA 


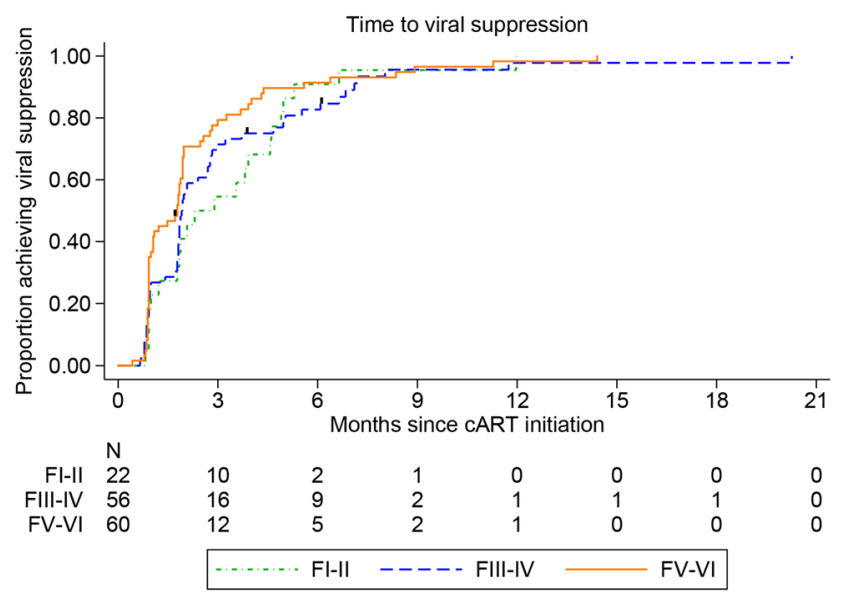

Figure 3 Kaplan-Meier survival curve of months from cART initiation to achieving viral suppression among The Netherlands Cohort Study on Acute HIV infection cohort study participants stratified by Fiebig stage, August 2015 to July 2020. Viral suppression was defined as the first documented viral load $<40$ copies $/ \mathrm{mL}$. The black hashes represent censored participants. Two participants with missing viral load values were excluded from this analysis. cART, combination antiretroviral therapy; F., Fiebig stage.

testing if a person presents with exposure to HIV in the previous 3 months combined with AEHI symptoms. For these reasons, it may be more likely that AEHI is identified in a person with symptoms than in a person without symptoms. Furthermore, due to the high HIV incidence in MSM and routine screening offered to MSM and other high-incidence populations, it is more likely to identify AEHI in these groups than in low-incidence populations, such as heterosexual individuals.

\section{Fiebig staging}

According to the study protocol, participants are enrolled within 24 hours of diagnosis and samples are obtained on the day of enrolment. Subsequently, cART should be initiated within 24 hours of enrolment. However, in some cases, there may be a delay between diagnosis and enrolment or between enrolment and cART initiation due to logistical factors (eg, study staff not being available for enrolment during weekends) or participant-related factors (eg, a participant needing time to decide whether they wish to initiate cART immediately). Fiebig stage at cART initiation (rather than at diagnosis) is correlated with the size of the HIV reservoir and the Fiebig stage may evolve rapidly between diagnosis and start of cART and study enrolment ${ }^{2131}$ Therefore, in these cases, we determine Fiebig stages on samples obtained on the day of cART initiation or, if such a sample is not available, within 3 days before or after cART initiation. For participants of whom no sample is available within this time frame, the Fiebig stage is estimated by extrapolation based on the estimated duration of each stage as described by Fiebig et al (Fiebig stage I: 5 days; II: 5 days; III: 3 days; IV 6 days; V: 70 days; VI: open ended) ${ }^{22}$ : for example, if a sample has been obtained 6 days before cART initiation and indicated Fiebig stage IV, we classify this participant as Fiebig stage $\mathrm{V}$ at cART initiation.

\section{Treatment}

Consenting participants start cART within 24 hours of enrolment with a regimen including emtricitabine/tenofovir disoproxil fumarate $200 / 245 \mathrm{mg}$ once daily, dolutegravir (DTG) $50 \mathrm{mg}$ once daily and darunavir/ritonavir (DRV/r) 800/100 mg once daily. DRV/r was added to standard triple-drug treatment to account for potential transmitted HIV drug resistance, while awaiting the results of genotypic resistance testing. DRV/r was selected because of the virtual lack of transmitted resistance to HIV protease inhibitors in the Netherlands, its high genetic barrier and good tolerability. ${ }^{32}$ In all participants, $\mathrm{DRV} / \mathrm{r}$ is discontinued at week 4 depending on the results of baseline resistance testing. From 2015 through 2019, participants used DTG two times per day during these first 4 weeks of treatment, and plasma samples to evaluate DTG and $\mathrm{DRV} / \mathrm{r}$ pharmacokinetics were collected, as DRV/r could potentially decrease plasma levels of DTG. ${ }^{34}$ Since 2020, the protocol was adjusted to DTG $50 \mathrm{mg}$ once daily, as a dose adjustment for DTG is no longer recommended when used with $\mathrm{DRV} / \mathrm{r}^{35}$ We have ensured continued pharmacokinetic analysis of DTG before and after DTG dose adjustment. ${ }^{36}$ The HIV treating physicians and a specialised HIV nurse perform HIV counselling with respect to readiness to initiate cART, cART adherence, quality of life and sexual behaviour at enrolment and throughout the study period. Data are collected in collaboration with AIDS Therapy Evaluation in the Netherlands (ATHENA) National HIV cohort, which encompass data of $98 \%$ of all PLWH in care in the Netherlands. ${ }^{37}$ Clinical data in the ATHENA cohort are collected prospectively by trained data monitors using standardised case record forms.

\section{Patient and public involvement}

The NOVA cohort study has a strong engagement with PLWH as well as individuals behaviourally vulnerable for acquiring HIV. First, an essential element in the design of the NOVA cohort study has been to create awareness for AEHI among MSM, by launching two communication campaigns focusing on AEHI, its symptoms and the benefits of immediate treatment (www.hebikhiv. $\mathrm{nl} / \mathrm{en})$. These campaigns have been developed and delivered through cocreation with MSM living with and without HIV, through MSM focus groups, communication experts from the MSM community and the Dutch HIV Association. ${ }^{38}$ Second, the Dutch HIV Association of PLWH (Hiv Vereniging) is represented among the NOVA collaborators and provided with regular updates on progress of the study as well as scientific development on HIV cure. Finally, future clinical trials of novel interventions aiming at achieving post-treatment control will likely include analytic treatment interruption (ATI) and thereby can be expected to have emotional and physical 
A
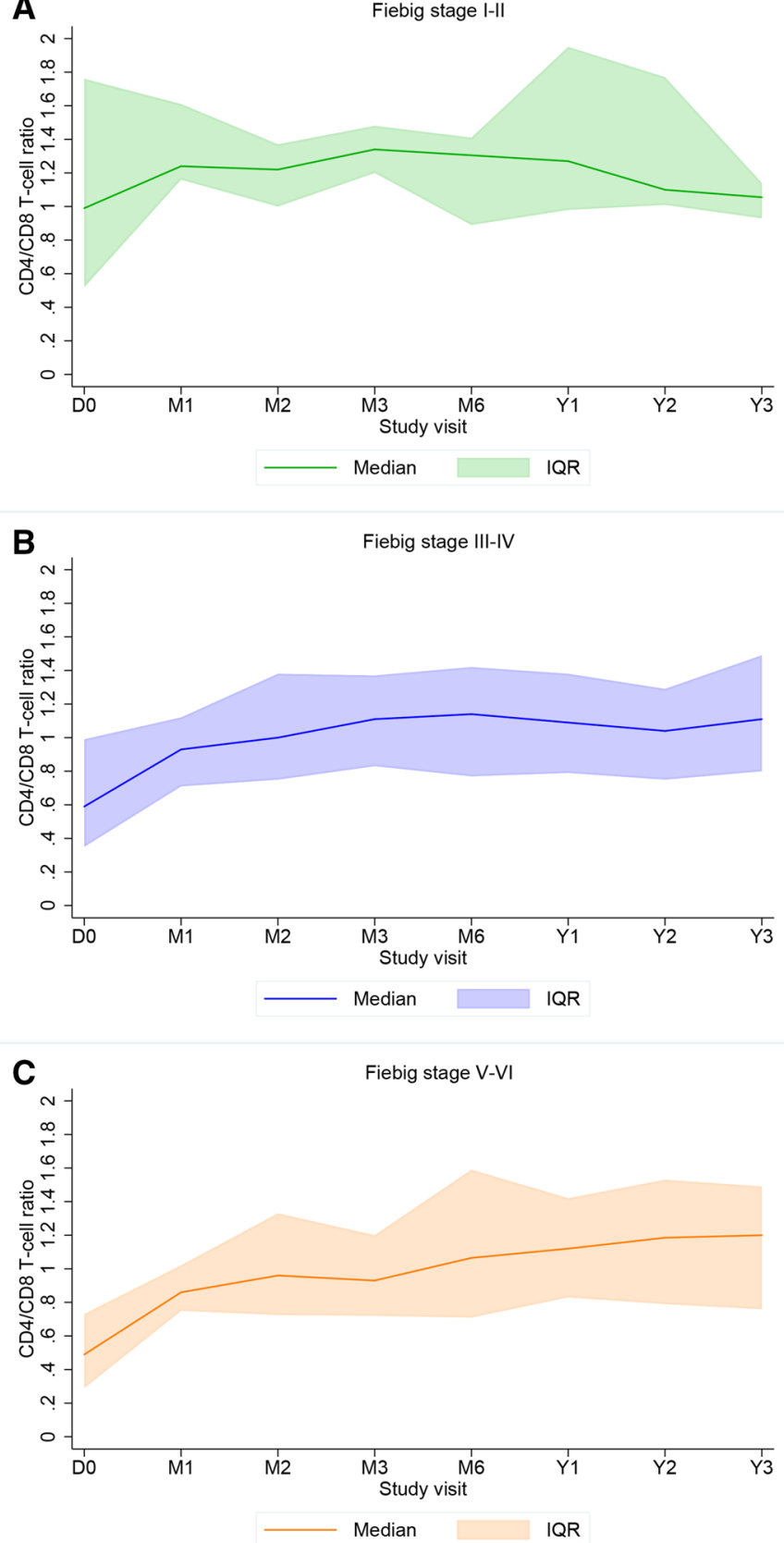

Figure 4 Median CD4/CD8 T-cell ratio and IQR among The Netherlands Cohort Study on Acute HIV infection cohort study participants, August 2015 to July 2020. (A) Displays CD4/CD8 T-cell ratio median and IQR among participants who initiated cART during Fiebig stages I-II; (B) Fiebig stages III-IV; and (C) Fiebig stages V-VI. D, day ; M, month; Y, year.

impact on participants. ${ }^{39}$ Assessing the views of potential participants concerning ATI during cure trials is therefore essential and will provide indispensable information for trial design. We have recently explored knowledge and perception of HIV cure and willingness to participate in cure trials among NOVA participants by conducting 20 in-depth interviews. ${ }^{40}$ Furthermore, as part of this substudy, we explored barriers to undergo additional sampling in NOVA (such as in study groups 2 and 3).
Time from diagnosis to viral suppression

Starting cART during AEHI improves clinical outcomes. ${ }^{41}{ }^{42}$ Furthermore, initiation of cART during AEHI results in rapid plasma viral suppression and limits the size of the viral reservoir measured as HIVDNA in PBMC. ${ }^{21} 2243-45$ However, diagnosis of AEHI can be challenging and referral to an HIV treatment centre may cause delay in cART initiation. ${ }^{46} 47$ To overcome these barriers, an AEHI search and treat-to-suppression strategy was implemented at the STI clinic in Amsterdam in August 2015. In this strategy, mobilisation for AEHI testing through an AEHI communication campaign was combined with point-of-care HIV-RNA testing by RT-PCR, same-visit delivery of HIV diagnosis and same-day referral to an HIV treatment centre for immediate cART initiation. This strategy proved to be a feasible and effective approach in diagnosing AEHI and significantly reducing the time from diagnosis to viral suppression. ${ }^{48}$ The HIV treatment centres in Rotterdam work together to liaise with STI clinics and general practitioners and have direct access to point-of-care HIV-RNA testing by RT-PCR to facilitate referring people diagnosed with AEHI and start cART within 24 hours. To facilitate early diagnosis, we implemented education on AEHI and NOVA in regional meetings with general practitioners, STI clinics and firstline laboratories and made point-of-care HIV-RNA testing available for them. Recently, an online platform was launched for infectious diseases specialists, general practitioners and physicians working at STI clinics to enable direct communication and potentially facilitating faster referral of people diagnosed with AEHI in the future.

\section{Findings to date}

\section{Baseline characteristics}

The NOVA cohort study was initiated in August 2015 and since then (data update July 2020) 140 participants have been enrolled. Of these, $131(93.6 \%)$ remain in active follow-up. Overall, 76 (54.3\%) participants have been included in group 1; 55 (39.3\%) in group 2; and 9 (6.4\%) in group 3 (figure 2). Barriers for the decision to participate in study group 2 or 3 explored in 20 in-depth interviews included fear of possible risks related to sampling procedures (such as lumbar puncture or intestinal biopsy), needle phobia, being overwhelmed by the HIV diagnosis and practical concerns, such as the difficulty to combine study visits with work. The majority of participants are men and reported to be MSM (124, 92.5\%). Further baseline characteristics are provided in table 1. On average, approximately 30 participants have been enrolled each year, with the exception of 2020, during which HIV testing, diagnosis and NOVA enrolment was strongly reduced due to the COVID-19 pandemic. ${ }^{49}$ Median duration of follow-up is 2.4 (IQR 1.4-3.1) years. The distribution of participants among Fiebig stages is as follows: Fiebig I, 3 (2.1\%); II, 20 (14.3\%); III, 7 (5.0\%); IV, 49 (35.0\%); V, 39 (27.9\%); VI, 22 (15.7\%). For seven $(5.0 \%)$ participants, no sample had been obtained 3 days before or after cART initiation and the Fiebig stage is 


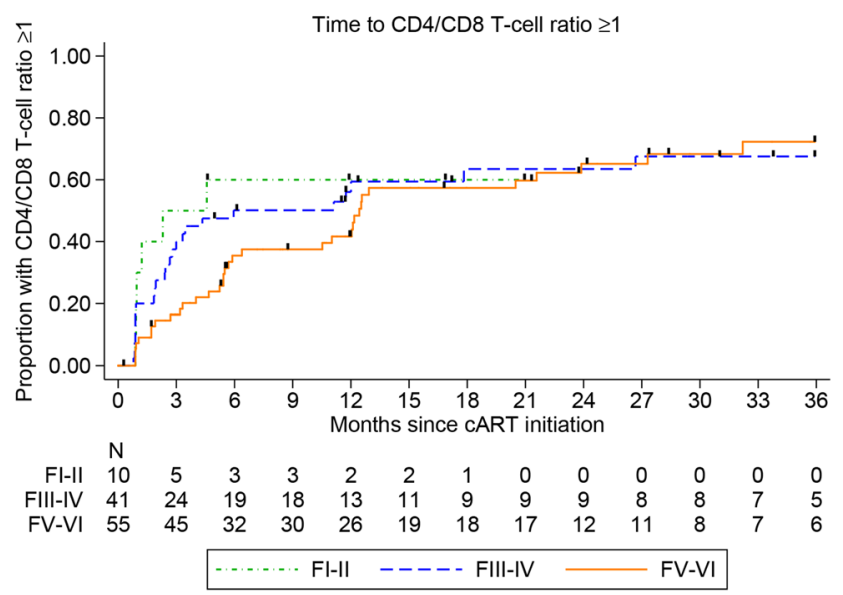

Figure 5 Kaplan-Meier survival curve of months from cART initiation to reaching a CD4/CD8 T-cell ratio $\geq 1$ among The Netherlands Cohort Study on Acute HIV infection cohort study participants with a baseline CD4/CD8 T-cell ratio $<1$, stratified by Fiebig stage, August 2015 to July 2020. The black hashes represent censored participants. The following participants were excluded from this analysis: one participant with missing CD4/CD8 T-cell ratio values, one participant with only one value available, which was obtained before CART initiation and 32 participants with a CD4/CD8 T-cell ratio $\geq 1$ at baseline. cART, combination antiretroviral therapy; F, Fiebig stage.

estimated by extrapolation based on the estimated duration of each stage.

Participants in the NOVA cohort study initiated cART a median of 1 (IQR 0-7) day after HIV diagnosis. No resistance-associated mutations relevant to the cART regimen provided in NOVA were detected at baseline. Median plasma HIV RNA at baseline was 6.4 (IQR 5.0-7.0) $\log 10$ copies/mL among participants with Fiebig stages I-II, 6.4 (IQR 5.5-7.0) among those with stages III-IV and 5.0 (IQR 4.5-5.9) among those with stages V-VI (table 2). Median time from cART initiation to viral suppression was slightly longer among participants treated during Fiebig stages I-II (10.0 (IQR 5.3-20.0) weeks) than among participants treated during stages $\mathrm{V}-$ VI (7.7 (IQR 4.0-12.0) weeks) (figure 3).

\section{Time to CD4/CD8 T-cell ratio of at least 1}

Median CD4 ${ }^{\text {pos }}$ T-cell count at baseline was 500 (IQR $330-580)$ cells $/ \mathrm{mm}^{3}$ among Fiebig stages I-II participants, 480 (IQR 325-620) among stages III-IV participants and 560 (IQR 422-730) among stages V-VI participants. PrecART CD4/CD8 T-cell ratio $<1$ is associated with chronic immune activation among PLWH who initiated treatment during chronic stages of infection ${ }^{50}$ and is inversely correlated with the size of the viral reservoir among treated people with AEHI. ${ }^{52}$ Therefore, we assessed the time to the first $\mathrm{CD} 4 / \mathrm{CD} 8 \mathrm{~T}$-cell ratio of $\geq 1$ in our cohort stratified by Fiebig stage, using Kaplan-Meier estimates. Longitudinal $\mathrm{CD} 4^{\text {pos }}$ T-cell and $\mathrm{CD} 8^{\text {pos }}$ T-cell results were available from 139 (enrolment), 70 (month $1, \pm 15$ days), 55 (month $2, \pm 15$ days), 61 (month $3, \pm 15$ days), 88 (month $6, \pm 30$ days), 90 (year $1, \pm 60$ days), 51 (year $2, \pm 60$ days) and 39 (year $3, \pm 60$ days) participants. Median CD4/ CD8 T-cell ratio at enrolment was 0.55 (IQR 0.37-0.96), with $32(23.0 \%)$ participants having a ratio $\geq 1$, including all $3(9.4 \%)$ who had initiated cART during Fiebig stage I. Figure 4 displays median CD4/CD8 T-cell ratios during the first 3 years of follow-up. Among participants with a CD4/CD8 T-cell ratio $<1$ at enrolment, median time to


weeks. This was shorter among participants treated during Fiebig stages I-II ( $\mathrm{n}=10 ; 10.0$ weeks) than among those treated during stages III-IV ( $\mathrm{n}=41 ; 25.9$ weeks) or during stages V-VI ( $\mathrm{n}=55$; 54.0 weeks; figure 5$)$. However, as only 10 participants with Fiebig stages I-II had a CD4/CD8 T-cell ratio $<1$ at cART initiation, these findings should be interpreted with caution.

In 2015, we established the NOVA as an ongoing multicentre prospective cohort study of people with AEHI in the Netherlands. Since then, we have been enrolling an average of approximately 30 participants per year, with the exception of 2020 during which enrolment was strongly reduced due to the COVID-19 pandemic. The high retention of $94 \%$ and a median of 8 weeks to viral suppression suggest that starting cART during AEHI is acceptable and adherence to cART is high. The considerable amount of time spent on counselling by a specialised HIV nurse and treating physicians within the context of the NOVA could be a factor influencing these findings. Alternatively, PLWH willing to participate in the study may be more motivated compared with those who refused to take part. Unfortunately, we were not able to collect data on PLWH not willing to participate.

In the years since start, we have been able to enrol participants in the early Fiebig stages I and II. This is particularly challenging due to the short duration of these stages. ${ }^{29}$ The challenge to rapidly enrol participants diagnosed with Fiebig stages I and II has been described by others, and a delay between diagnosis and enrolment often results in participants no longer being in this phase of infection at enrolment. ${ }^{31}$ We will continue to focus on accelerated diagnostic and referral pathways in collaboration with STI clinics, general practitioners and hospitals in order to increase the number of participants in these early infection stages. Unfortunately, for $5 \%$ of participants, there was no sample available in the window 3 days before or after cART initiation, and we used an estimated Fiebig stage rather than a measured Fiebig stage for these participants. This strategy may have the potential to introduce error, as the duration of each stage can vary from person to person..$^{29}$ In future studies within this cohort, we will conduct sensitivity analyses excluding participants with an estimated Fiebig stage. Furthermore, we once more emphasised to the study sites that future participants should be enrolled within 24 hours of diagnosis and samples are obtained on the day of enrolment, and we have planned regular investigator meetings to support this practice. 
Together now almost half of the total cohort participates in the 'extended' groups 2 and 3 . The number of participants enrolled in group 3 is limited, and we may not have sufficient power to perform analyses within this group stratified by Fiebig stage. However, the number and material will be sufficient and valuable for longitudinal studies within the same participant, for example, to study viral reservoir dynamics, including within host viral evolution. In addition, we will continue our efforts to enrol participants in group 3 in the coming years.

The blood and tissue samples allow us to longitudinally characterise the viral reservoir and the properties of the HIV-specific immune response in great detail. We plan to comprehensively characterise the viral reservoir with respect to proviral latency and residual replication competence; and characterisation of the cellular localisation of the viral reservoir with respect to type of cell and cellular phenotype (including activation status). The HIV-specific immune response will be characterised by assessing the frequency and breadth of HIV-specific CD8 ${ }^{\text {pos }} \mathrm{T}$ cells, the phenotype and functional properties of the HIV-specific $\mathrm{CD} 8^{\text {pos }} \mathrm{T}$ cells and the distribution of $\mathrm{CD} 8^{\mathrm{pos}} \mathrm{T}$ cells in lymph nodes versus peripheral blood. Furthermore, in the coming years, we expect novel approaches to HIV cure to be developed, for example, the in vivo efficacy of various approaches to purge the latent viral reservoir and new ex vivo studies investigating the response of latency reversing agents and immune-based therapies in various cell types from PLWH who are on cART. ${ }^{53}$ NOVA will provide a valuable platform to conduct clinical trials evaluating such newly developed approaches, also in light of existing and future international collaborations. ${ }^{54}$

The NOVA cohort study complements several other prospective cohorts on AEHI worldwide (among others those from US, African, South American and Thai sites NCT00296660 and NCT02859558; San Francisco NCT02656511; Gent NCT03449706; Zurich NCT00537966; Bangkok RV254/SEARCH010 studies ${ }^{21}$; East Africa and Thailand RV21 $7^{55}$; and the South African FRESH cohort). ${ }^{28}$ The course of HIV infection (including viral load at AEHI, viral setpoint and viral reservoir) is highly variable between cohorts. ${ }^{56-58}$ This variation is in part determined by age, sex, mode of HIV transmission and, importantly, geographical location and HIV subtype (B vs non-B). ${ }^{57} 58$ Differences in level of viremia during AEHI and viral setpoint have been shown to be influenced by host genetic and viral factors. ${ }^{569}$ An important host genetic factor is HLA type, which is differently distributed across geographic (ethnic) regions. ${ }^{57} \mathrm{~A}$ viral factor that may determine the course of infection includes clade. A post-hoc analysis by Omondi et al indeed showed a difference in viral reservoir size between a Ugandan (black) and US (white) cohort infected with different viral clades. ${ }^{58}$ Because these determinants of HIV infection may impact formation of reservoir and immune response, we need multiple cohorts from around the world and different geographies to study this.

The nine Dutch HIV treatment centres that participate in NOVA cover the areas of the Netherlands known to have the highest HIV prevalence and serving $65 \%$ of all
PLWH in care. The vast majority of new HIV diagnoses in the Netherlands occur among MSM. ${ }^{32}{ }^{60}$ This may explain the over-representation of MSM (93\%) in NOVA. Thus far, approximately $35 \%$ of MSM diagnosed with AEHI in the Netherlands have been enrolled in NOVA. ${ }^{32}$ We expect to increase this proportion in the coming years as participating study sites were added over a period of years and some of these sites have started to enrol participants only recently.

In conclusion, the NOVA cohort study is a well characterised nationwide cohort of people who initiated cART during AEHI and provides a unique platform to conduct detailed analyses of the HIV reservoir and the host immune response and future interventional studies aimed at achieving durable HIV control in the absence of cART and cure.

\section{Author affiliations}

${ }^{1}$ Department of Infectious Diseases, Public Health Service of Amsterdam, Amsterdam, Netherlands

${ }^{2}$ Department of Internal Medicine, Division of Infectious Diseases, Amsterdam Institute for Infection and Immunity (All), Amsterdam UMC, University of Amsterdam, Amsterdam, Netherlands

${ }^{3}$ Department of Internal Medicine, Division of Infectious Diseases, Erasmus University Medical Center, Rotterdam, Netherlands

${ }^{4}$ HIV Monitoring Foundation, Amsterdam, Noord-Holland, Netherlands

${ }^{5}$ Department of Global Health, Amsterdam Institute for Global Health and

Development, Amsterdam UMC, University of Amsterdam, Amsterdam, the

Netherlands

${ }^{6}$ Department of Viroscience, Erasmus University Medical Center, Rotterdam, Netherlands

${ }^{7}$ Department of Internal Medicine, Amsterdam University Medical Centre, Amsterdam, Netherlands

Acknowledgements We would kindly like to thank all participants of the The Netherlands Cohort Study on Acute HIV infection (NOVA) cohort study. We would also like to thank the members of the Erasmus MC HIV Eradication Group, all medical doctors, research nurses and laboratory personnel involved in the NOVA cohort study at the Amsterdam University Medical Centre, the Erasmus University Medical Center, Maasstad Hospital Rotterdam, Onze Lieve Vrouwe Gasthuis, DC Klinieken, UMC Utrecht, Radboud UMC, Leiden UMC and Rijnstate Hospital for enrolment, follow-up and (handling of) sampling of NOVA participants, including Karin Grintjes, Reinout van Crevel, Janette Rahamat-Langendoen, Henk Scheper, Jutte de Vries, Annouschka Weijsenfeld, Frank Pijnappel, Agnes Harskamp, Neeltje Kootstra, Suzanne Jurriaans, Marc van der Valk, Hans-Erik Nobel, Arne van Eeden, Loek Elsenburg, Kees Brinkman, Imke Hooijenga, Janneke Stalenhoef, Mark Claassen, Petra van Bentum, Jan den Hollander, Jeroen van Kampen, Yvonne Muller, Peter Katsikis, Tokameh Mahmoudi, Rob Gruters, Andy Hoepelman, Monique Nijhuis, Anne Wensing. We would also like to thank Stichting HIV Monitoring for providing data collection and management support, including Mariska Hillebregt, Ard van Sighem and Leonie de Groot-Berndsen, and the Dutch HIV association (Hiv Vereniging) for providing continuous study support and involvement, including Bertus Tempert and Renee Finkenflügel. CB Our friend and colleague sadly passed away on 26 February 2021.

Collaborators The Netherlands Cohort Study on Acute HIV infection (NOVA) cohort study currently collaborates with several research groups in the Netherlands and enrols participants from nine clinical HIV treatment centres, and aims to actively engage additional Dutch study sites. The NOVA cohort study is overseen by a governing board composed of representatives of the two coordinating sites, Amsterdam UMC, Academic Medical CenterCentre site and Erasmus University Medical Centre. For further information visit the H-TEAM web page (https://hteam. $\mathrm{nl} /$ ?lang=en).

Contributors GdB, CR, JMP, PR, CB and AV designed the study. MD and HP collaborated with Stichting HIV Monitoring Netherlands to collect data. MD conducted the statistical analysis. MD and HP drafted the manuscript which was revised by JMP, PR, CB, AV, CR and GdB. GdB and CR supervised the project and are also responsible for its continued management. All authors reviewed, critically revised and approved the manuscript. CR contributed equally as last authors. GJB acts as the guarantor. 
Funding The H-TEAM initiative is being supported by Aids Fonds (grant number: 2013169), Stichting Amsterdam Dinner Foundation, Bristol-Myers Squibb International Corp. (study number: Al424-541), Gilead Sciences Europe Ltd (grant number: PA-HIV-PREP-16-0024), Gilead Sciences (protocol numbers: CO-NL276-4222, C0-US-276-1712), M.A.C AIDS Fund.

Competing interests GdB has received grants through her institution from Bristol-Meyer Squibbs and Mac Aids Fund; honoraria to her Institution for scientific advisory board participations for Gilead Sciences and speaker fees from Gilead Sciences and Takeda. PR has received grants through his institution from Gilead Sciences, Janssen Pharmaceutica, ViiV Healthcare and Merck; honoraria to his institution for scientific advisory board participation for Gilead Sciences, ViiV Healthcare, Merck and Teva. CR has received study grants from AIDSfonds, ZonMW, Dutch Federation Medical Specialists, Merck, Janssen-Cilag, Gilead and ViiV Healthcare. All other authors declared no conflicts of interest.

Patient and public involvement Patients and/or the public were involved in the design, or conduct, or reporting, or dissemination plans of this research. Refer to the Cohort description section for further details.

\section{Patient consent for publication Not applicable.}

Ethics approval Study participants provide written informed consent. The study has been approved by the medical ethical committee of the Amsterdam University Medical Centers (Academic Medical Center site) (NL51613.018.14)

Provenance and peer review Not commissioned; externally peer reviewed.

Data availability statement Data are available upon reasonable request. We endeavour to make the data used in any The Netherlands Cohort Study on Acute HIV infection (NOVA) manuscript publicly available, within the limits of the ethical governance under which the data were collected. To this end, we will share data directly with interested parties for two purposes: (1) verification and replication of an already published analysis derived from NOVA, (2) novel scientific research projects using NOVA data. To facilitate this, requests for data sharing can be made on a case-by-case basis following submission of a concept sheet. Once submitted the proposed research/analysis will undergo review by the NOVA team for evaluation of the scientific value, relevance to the study, design and feasibility, statistical power and overlap with existing projects. If the proposed analysis is for verification/replication, data will then be made available. If the proposed research is for novel science, upon completion of the review, feedback will be provided to the proposer(s). In some circumstances, a revision of the concept may be requested. If the concept is approved for implementation, a writing group will be established consisting of the proposers (up to three people that were centrally involved in the development of the concept) and members of the NOVA study group (or other appointed cohort representatives). All people involved in the process of reviewing these research concepts are bound by confidentiality. For more information about the procedure, data sharing or collaboration in general, please contact dr. GdB: g.j. debree@amsterdamumc.nl.

Open access This is an open access article distributed in accordance with the Creative Commons Attribution Non Commercial (CC BY-NC 4.0) license, which permits others to distribute, remix, adapt, build upon this work non-commercially, and license their derivative works on different terms, provided the original work is properly cited, appropriate credit is given, any changes made indicated, and the use is non-commercial. See: http://creativecommons.org/licenses/by-nc/4.0/.

\section{ORCID iDs}

Maartje Dijkstra http://orcid.org/0000-0003-2561-7659

Henrieke Prins http://orcid.org/0000-0002-1452-7271

\section{REFERENCES}

1 Joint United nations programme on HIV/AIDS (UNAIDS). Ending the AIDS epidemic fact sheet - World AIDS Day, 2020. Available: https:// www.unaids.org/en/resources/fact-sheet

2 Havlir DV, Balzer LB, Charlebois ED, et al. Hiv testing and treatment with the use of a community health approach in rural Africa. $N$ Engl $J$ Med 2019;381:219-29.

3 Hayes RJ, Donnell D, Floyd S, et al. Effect of Universal testing and treatment on HIV Incidence - HPTN 071 (PopART). N Engl J Med 2019;381:207-18.

4 Sweeney SM, Vanable PA. The association of HIV-related stigma to HIV medication adherence: a systematic review and synthesis of the literature. AIDS Behav 2016;20:29-50.

5 van Bilsen WPH, Zimmermann HML, Boyd A, et al. Burden of living with HIV among men who have sex with men: a mixed-methods study. Lancet HIV 2020;7:e835-43.
6 Langebeek N, Kooij KW, Wit FW, et al. Impact of comorbidity and ageing on health-related quality of life in HIV-positive and HIVnegative individuals. AIDS 2017;31:1471-81.

7 Ndung'u T, McCune JM, Deeks SG. Why and where an HIV cure is needed and how it might be achieved. Nature 2019;576:397-405

8 Sáez-Cirión A, Bacchus C, Hocqueloux L, et al. Post-treatment HIV-1 controllers with a long-term virological remission after the interruption of early initiated antiretroviral therapy ANRS VISCONTI study. PLoS Pathog 2013;9:e1003211.

9 Kroon EDMB, Ananworanich J, Pagliuzza A, et al. A randomized trial of vorinostat with treatment interruption after initiating antiretroviral therapy during acute HIV-1 infection. J Virus Erad 2020;6:100004.

10 , Lundgren JD, Babiker AG, et al, INSIGHT START Study Group. Initiation of antiretroviral therapy in early asymptomatic HIV infection. N Engl J Med 2015;373:795-807.

11 Whitney JB, Hill AL, Sanisetty S, et al. Rapid seeding of the viral reservoir prior to SIV viraemia in rhesus monkeys. Nature 2014;512:74-7.

12 Valcour V, Chalermchai T, Sailasuta N, et al. Central nervous system viral invasion and inflammation during acute HIV infection. J Infect Dis 2012;206:275-82.

13 Bruner KM, Murray AJ, Pollack RA, et al. Defective proviruses rapidly accumulate during acute HIV-1 infection. Nat Med 2016;22:1043-9.

14 Ho Y-C, Shan L, Hosmane NN, et al. Replication-competent noninduced proviruses in the latent reservoir increase barrier to HIV-1 cure. Cell 2013;155:540-51.

15 Perreau M, Levy Y, Pantaleo G. Immune response to HIV. Curr Opin HIV AIDS 2013;8:333-40.

16 Chomont N, El-Far M, Ancuta P, et al. Hiv reservoir size and persistence are driven by $T$ cell survival and homeostatic proliferation. Nat Med 2009;15:893-900.

17 Chun TW, Carruth L, Finzi D, et al. Quantification of latent tissue reservoirs and total body viral load in HIV-1 infection. Nature 1997;387:183-8.

18 Finzi D, Hermankova M, Pierson T, et al. Identification of a reservoir for HIV-1 in patients on highly active antiretroviral therapy. Science 1997;278:1295-300.

19 Persaud D, Gay H, Ziemniak C, et al. Absence of detectable HIV-1 viremia after treatment cessation in an infant. N Engl J Med 2013;369:1828-35

20 Hocqueloux L, Prazuck T, Avettand-Fenoel V, et al. Long-Term immunovirologic control following antiretroviral therapy interruption in patients treated at the time of primary HIV-1 infection. AIDS 2010;24:1598-601.

21 Ananworanich J, Chomont N, Eller LA, et al. Hiv DNA set point is rapidly established in acute HIV infection and dramatically reduced by early art. EBioMedicine 2016;11:68-72.

22 Ananworanich J, Schuetz A, Vandergeeten C, et al. Impact of multi-targeted antiretroviral treatment on gut $\mathrm{T}$ cell depletion and HIV reservoir seeding during acute HIV infection. PLoS One 2012; 7:e33948.

23 Vanham G, Buvé A, Florence E, et al. What is the significance of posttreatment control of HIV infection vis-à-vis functional cure? AIDS 2014;28:603-5.

24 Chun T-W, Justement JS, Moir S, et al. Decay of the HIV reservoir in patients receiving antiretroviral therapy for extended periods: implications for eradication of virus. J Infect Dis 2007;195:1762-4.

25 Archin NM, Vaidya NK, Kuruc JD, et al. Immediate antiviral therapy appears to restrict resting CD4+ cell HIV-1 infection without accelerating the decay of latent infection. Proc Natl Acad Sci U S A 2012;109:9523-8.

26 Colby DJ, Trautmann L, Pinyakorn S, et al. Rapid HIV RNA rebound after antiretroviral treatment interruption in persons durably suppressed in Fiebig I acute HIV infection. Nat Med 2018;24:923-6.

27 Takata H, Buranapraditkun S, Kessing C, et al. Delayed differentiation of potent effector $\mathrm{CD}^{+} \mathrm{T}$ cells reducing viremia and reservoir seeding in acute HIV infection. Sci Trans/ Med 2017;9. doi:10.1126/ scitransImed.aag1809. [Epub ahead of print: 1502 2017].

28 Ndhlovu ZM, Kazer SW, Nkosi T, et al. Augmentation of HIV-specific T cell function by immediate treatment of hyperacute HIV-1 infection. Sci Trans/ Med 2019;11. doi:10.1126/scitransImed.aau0528. [Epub ahead of print: 2205 2019].

29 Fiebig EW, Wright DJ, Rawal BD, et al. Dynamics of HIV viremia and antibody seroconversion in plasma donors: implications for diagnosis and staging of primary HIV infection. AIDS 2003;17:1871-9.

30 Dijkstra M, de Bree GJ, Stolte IG, et al. Development and validation of a risk score to assist screening for acute HIV-1 infection among men who have sex with men. BMC Infect Dis 2017;17:425.

31 Stekler JD, Tapia K, Maenza J, et al. No time to delay! Fiebig stages and referral in acute HIV infection: seattle primary infection program experience. AIDS Res Hum Retroviruses 2018;34:657-66. 
32 van Sighem Al, FWNM W, Boyd A, et al. Human Immonudeficiency virus (HIV) infection in the Netherlands. 2020. Amsterdam: Stichting HIV Monitoring, 2019.

33 Aoki M, Das D, Hayashi $\mathrm{H}$, et al. Mechanism of darunavir (DRV)'s high genetic barrier to HIV-1 resistance: a key V32I substitution in protease rarely occurs, but once it occurs, it predisposes HIV-1 to develop DRV resistance. mBio 2018;9. doi:10.1128/mBio.02425-17. [Epub ahead of print: 0603 2018].

34 Song I, Min SS, Borland J, et al. The effect of lopinavir/ritonavir and darunavir/ritonavir on the HIV integrase inhibitor S/GSK1349572 in healthy participants. J Clin Pharmacol 2011;51:237-42.

35 Hiv drug interactions, University of Liverpool. Available: https:// www.hiv-druginteractions.org/interactions/97705; [Accessed 29 Oct 2020]

36 Prins HA, Zino L, Svensson EM. Pharmacokinetics of dolutegravir combined with ritonavir boosted darunavir in treatment-naïve individuals enrolled in the Netherlands Cohort Study on Acute HIV infection (NOVA). Submitted 2021.

37 Boender TS, Smit C, Sighem Avan, et al. Aids therapy evaluation in the Netherlands (ATHENA) national observational HIV cohort: cohort profile. BMJ Open 2018;8:e022516.

38 Davidovich U, Dijkstra M, van Bijnen A. P4.105 Highly successful engagement in an acute hiv-infection (AHI) awareness campaign and intervention in amsterdam \& its yield of ahi diagnoses at the city's sti clinic. Sex Transm Infect 2017;93:A230.

39 Eyal N. The benefit/risk ratio challenge in clinical research, and the case of HIV cure: an introduction. J Med Ethics 2017;43:65-6.

40 Dijkstra M, Rennie S HLP. Perception and willingness to participate in HIV cure clinical trials among people who initiated treatment during acute HIV infection in Amsterdam. 23rd international AIDS conference, San Francisco: PEB0271, 2020.

41 Ford N, Migone $\mathrm{C}$, Calmy $\mathrm{A}$, et al. Benefits and risks of rapid initiation of antiretroviral therapy. AIDS 2018;32:17-23.

42 Grijsen ML, Steingrover R, Wit FWNM, et al. No treatment versus 24 or 60 weeks of antiretroviral treatment during primary HIV infection: the randomized Primo-SHM trial. PLoS Med 2012;9:e1001196.

43 Crowell TA, Phanuphak N, Pinyakorn S, et al. Virologic failure is uncommon after treatment initiation during acute HIV infection. AIDS 2016;30:1943-50.

44 Girometti N, Nwokolo N, McOwan A, et al. Outcomes of acutely HIV1 -infected individuals following rapid antiretroviral therapy initiation. Antivir Ther 2017;22:77-80.

45 Hoenigl M, Chaillon A, Moore DJ, et al. Rapid HIV viral load suppression in those initiating antiretroviral therapy at first visit after HIV diagnosis. Sci Rep 2016;6:32947.

46 Sudarshi D, Pao D, Murphy G, et al. Missed opportunities for diagnosing primary HIV infection. Sex Transm Infect 2008;84:14-16.
47 Pilcher CD, Ospina-Norvell C, Dasgupta A, et al. The effect of sameday observed initiation of antiretroviral therapy on HIV viral load and treatment outcomes in a US public health setting. $J$ Acquir Immune Defic Syndr 2017;74:44-51.

48 Dijkstra M, van Rooijen MS, Hillebregt MM, et al. Decreased time to viral suppression after implementation of targeted testing and immediate initiation of treatment of acute human immunodeficiency virus infection among men who have sex with men in Amsterdam. Clin Infect Dis 2021;72:1952-60.

49 Hensley KS, Jordans CCE, van Kampen JJA, et al. Significant impact of COVID-19 on HIV care in hospitals affecting the first Pillar of the HIV care continuum. Clin Infect Dis 2021. doi:10.1093/cid/ciab445. [Epub ahead of print: 16 May 2021]

50 Lu W, Mehraj V, Vyboh K, et al. CD4:CD8 ratio as a frontier marker for clinical outcome, immune dysfunction and viral reservoir size in virologically suppressed HIV-positive patients. J Int AIDS Soc 2015;18:20052.

51 Serrano-Villar S, Sainz T, Lee SA, et al. Hiv-Infected individuals with low CD4/CD8 ratio despite effective antiretroviral therapy exhibit altered $T$ cell subsets, heightened CD8 $+T$ cell activation, and increased risk of non-AIDS morbidity and mortality. PLoS Pathog 2014;10:e1004078

52 Hurst J, Hoffmann M, Pace M, et al. Immunological biomarkers predict HIV-1 viral rebound after treatment interruption. Nat Commun 2015;6:8495.

53 Pitman MC, Lau JSY, McMahon JH, et al. Barriers and strategies to achieve a cure for HIV. Lancet HIV 2018;5:e317-28.

54 Rokx C, Prins HAB, Vandekerckhove L, et al. Launching a multidisciplinary European collaboration towards a cure for HIV: the EU2Cure Consortium. J Virus Erad 2021;7:100045.

55 Robb ML, Eller LA, Rolland M. Acute HIV-1 infection in adults in East Africa and Thailand. N Engl J Med 2016;375:1195.

56 Bartha I, McLaren PJ, Brumme C, et al. Estimating the respective contributions of human and viral genetic variation to HIV control. PLoS Comput Biol 2017;13:e1005339.

57 Naranbhai V, Carrington M. Host genetic variation and HIV disease: from mapping to mechanism. Immunogenetics 2017;69:489-98.

58 Omondi FH, Chandrarathna S, Mujib S, et al. HIV subtype and Nefmediated immune evasion function correlate with viral reservoir size in Early-Treated individuals. J Virol 2019;93. doi:10.1128/JVI.0183218. [Epub ahead of print: 1503 2019]

59 Blanquart F, Wymant C, Cornelissen M, et al. Viral genetic variation accounts for a third of variability in HIV-1 set-point viral load in Europe. PLoS Biol 2017;15:e2001855.

60 Ratmann O, van Sighem A, Bezemer D, et al. Sources of HIV infection among men having sex with men and implications for prevention. Sci Transl Med 2016;8:320ra2. 